\title{
Implementación de la metodología ABP (Aprendizaje Basado en Problemas) en estudiantes de último año de la carrera de Nutrición
}

\begin{abstract}
Alejandro Martínez-Rodríguez ${ }^{\text {a }}$ Stephanie Torrijo-Boix ${ }^{b}$
${ }^{a}$ Departamento de Química Analítica, Nutrición y Bromatología. Facultad de Ciencias. Universidad de Alicante. amartinezrodriguez@ua.es y ${ }^{b}$ Departamento de Química Analítica, Nutrición y Bromatología. Facultad de Ciencias. Universidad de Alicante. stephanie.torrijo@ua.es
\end{abstract}

\section{Resumen}

La innovación docente en el aula cursa con involucrar al alumnado en el proceso enseñanza-aprendizaje. Los objetivos fueron favorecer el desarrollo de las competencias y objetivos educativos de la parte práctica de la asignatura de Nutrición Deportiva, así como comparar los resultados obtenidos con el alumnado del curso inmediatamente anterior, en relación con la participación en jornadas científicas y las calificaciones académicas. En este trabajo, se ha desarrollado la implementación de la metodología ABP (Aprendizaje Basado en Problemas) en el alumnado de cuarto curso del Grado en Nutrición Humana y Dietética. La evaluación de los cambios producidos en la implementación se realizaron mediante entrevistas al alumnado, registro de actividades y calificaciones académicas. Los resultados manifestaron que el alumnado que realizó la parte práctica de la asignatura mediante la metodología ABP presentaron una mayor participación en jornadas científicas, y obtuvieron mejores calificaciones en significativamente. Asimismo, también mostraron cambios favorables en el desarrollo de las competencias y objetivos educativos en materia de nutrición deportiva. A modo de conclusión, el alumado implementado con la metodología ABP, tuvieron consonancia en el conocimiento y aplicación de estrategias de resolución de problemas que puede ser empleada en el contexto profesional del dietista-nutricionista.

Palabras clave: competencias, innovación, formación, enseñanza. 


\section{Introducción}

Enmarcadas en el Espacio Europeo de Educación Supuerior (EEES), las instituciones, inmersas en un periodo de cambios en la oferta educativa (Michavila, 2009), apuestan por un proceso de formación de adquisición de las competencias necesarias por parte del alumnado (Mauri, 2008). Es por ello, que desde las universidades se promueven múltiples propuestas e iniciativas innovadoras que tratan de integrar nuevas herramientas y estrategias con el propósito de mejorar el proceso de enseñanza-aprendizaje (Salinas, 2004). Habitualmente, los cambios que se producen en la enseñanza a partir de los proyectos de innovación, se centran predominantemente en la utilización de nuevos materiales didácticos (García, 2010), sin embargo, para mejorar el aprendizaje del alumnado también se han de tener en cuenta tanto las tareas que se asignan, la agrupación del alumnado y la relación de este con el profesorado.

Según autores de referencia (Beraza, 2013), ponen de manifiesto que la innovación en docencia se basa en la aplicación de tres condiciones importantes, como son la apertura (cierta flexibilidad que permite un "compromiso activo"), la actualización (aplicación de los nuevos conocimientos y recursos en los sistemas de enseñanza) y la mejora. Aparte de esto, se ha de tener en cuenta en la innovación educativa tanto la documentación como la evaluación. En este sentido, la evaluación va a ser un factor determinante en la aplicación de cualquier acción de innovación docente. De hecho, no se puede entender como efectiva ninguna innovación docente que carezca de un adecuado proceso de evaluación(López Pastor, 2008). Ya que a partir de la evaluación, permite la realización de reajustes del proceso enfocados a mejorar.

En alusión a las nuevas metodologías, se pueden encontrar nuevos paradigmas del aprendizaje (Michavila, 2009), que fundamentan los cambios en las tecnologías educativas, que no solamente están centradas en aplicaciones informáticas o comunicativas. Aunque hay que destacar la facilidad en la consulta de documentación que aporta la utilización de dispositivos digitales, y que cada vez tienen mayor presencia, tanto dentro como fuera del aula, frente a los convencionales (Singer, 2017). Esto no compite con que el nuevo modelo educativo se centra especialmente al estudiante (Michavila, 2009), y trata de buscar un refuerzo en la formación práctica, que sirva de sustento en la adherencia y consecución de los objetivos educativos (Peiteado, 2013) y competencias del alumnado (Cabra, 2008).

En la búsqueda del desarrollo de las competencias, se ha demostrado que la interacción con otros es uno de los factores que potencia el mismo (Cabra, 2008; Jofré, 2013). En relación con ello, el Método de Aprendizaje Basado en Problemas (ABP) se puede aplicar en el aula como nueva estrategia metodológica (Jofré, 2013). La ABP se basa en la identificación de un problema, que deberá ser analizado y sintetizado, para después de formular una 
hipótesis, permita poder elegir la estrategia que aporte una mejor solución al problema. Asimismo, también permite incorporar el aprendizaje colaborativo (Cuéllar, 2010), ya que la responsabilidad de la resolución del problema pasa por la cooperación entre los miembros del grupo, con los aspectos positivos que esto también conlleva dentro del proceso enseñanza-aprendizaje (interdependencia positiva, interacciones cara a cara, desarrollo de habilidades sociales y auto-reflexión en grupo). Mediante la ABP el alumnado ha demostrado mejora de diferentes habilidades como pueden ser la capacidad de análisis o síntesis. Y que además promueve el cambio de rol entre el profesor y el alumno, que entra a formar parte del proceso enseñanza-aprendizaje como protagonista (Gálvez, 2007).

Dentro de los desafíos que se plantean durante el proceso de enseñanza-aprendizaje, son diversas las variables que pueden influir en el mismo, como son el entorno (centro de estudios, materiales) o el tiempo (cuando el alumnado desarrolla las actividades) (Salinas, 2004). A partir de ahora, la formación de los docentes y la asunción de los nuevos modelos educativos, se convierte en otro de los factores importantes del proceso de enseñanzaaprendizaje (Esteban, 2005). En el que tienen que estar cada vez más formados, actualizados en las últimas evidencias empíricas (Novak, 2017), y tener la capacidad de influir y mediar con el alumnado (Warren, 2017). De este modo, se produce una adaptación del docente como "única fuente de formación o información" hacia a ser un orientador para desempeño de tareas y utilización de herramientas o recursos de forma atractiva (Warren, 2017); y que sea el propio alumnado quiénes puedan conformar su propio conocimiento (Gálvez, 2007).

En definitiva, las propuestas de innovación de la docencia universitaria llevan asociadas a una mejorar de la calidad de la docencia, y que puede ser justificada mediante los resultados de aprendizaje del alumnado. Además, como sugieren expertos en el área, el estudio y análisis del proceso innovador, es motivo de investigación continuada (Mauri, 2008). Por todo ello, la hipótesis del presente trabajo de innovación docente se basa en que la aplicación del ABP en el alumnado de último curso del Grado en Nutrición Humana y Dietética, puede contribuir favorablemente al proceso de enseñanza-aprendizaje, aumentar el compromiso del alumnado con la materia y mejorar las calificaciones académicas.

\section{Objetivos}

El objetivo general de la implementación es desarrollar la parte prática de la asignatura de Nutrición Deportiva de $4^{\circ}$ del Grado en Nutrición Humana y Dietética en base al método de Aprendizaje Basado en Problemas. Así como favorecer el desarrollo de habilidades y destrezas sociales, actitudinales y cognitivas para la búsqueda, análisis, integración y transferencia de conocimientos.

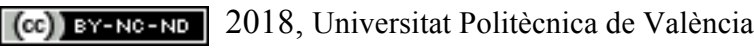


Los objetivos específicos son: a) Incorporar la metodología de Aprendizaje Basado en Problemas como estrategia transversal en las prácticas de Nutrición Deportiva; b) Desarrollar estrategias y métodos que permitan ser aplicados y transferidos en el futuro a situaciones semejantes para la resolución de problemas; c) Favorecer el aprendizaje autodirigido y el desarrollo de habilidades de comunicación en los estudiantes; d) Desarrollar la capacidad de trabajar en equipo y en redes a través de la posesión de actitudes y disposiciones que incluyen la flexibilidad y adaptabilidad; e) Estimular el pensamiento crítico en los estudiantes; d) Comparar los resultados de la participación en jornadas científicas y calificaciones académicas con el alumnado del curso anterior.

\section{Desarrollo de la innovación}

\subsection{Participantes}

En este estudio participaron estudiantes de cuarto curso del Grado en Nutrición Humana y Dietética, de la Universidad de Alicante, matriculados en la asignatura de Nutrición en la Actividad física y el Deporte. Esta asignatura es cuatrimestral y de carácter optativo, con una carga lectiva de 6 créditos ECTS, de los cuáles 1,2 son créditos ECTS prácticos. El número de estudiantes del curso 2016-2017 fue de 30, mientras que en 2017-2018 fueron 35 los estudiantes matriculados.

\subsection{Desarrollo de la innovación}

La implementación de la metodología ABP del proyecto, se basa en una metodología cualitativa de resolución de problemas. En esta, se emplea un problema definido como base para la orientación del alumnado durante el proceso de aprendizaje (Gálvez, 2007). Esta metodología de elaboración, análisis y respuesta o solución al problema, tiene como punto inicial un problema parecido al que el alumnado tendrá que dar respuesta en un futuro como profesional. En este sentido, se han de emplear los conocimientos previos adquiridos durante todo el proceso de formación previo $\left(1^{\circ}-3^{\circ}\right.$ curso). Para ello, tendrán que integrar los conocimientos de diferentes áreas y asignaturas, que darán como resultado una comprensión global y además serán capaces de detectar sus necesidades para dar solución al problema. Asimismo, tendrán a sus disposición todos los medios que consideren oportunos para ello, con la intención de garantizar la ayuda educativa ajustada al desarrollo de la autonomía y de la autorregulación del aprendizaje del alumno.

El problema inicial que se planteó fue la valoración de los hábitos alimentarios y el rendimiento deportivo en un grupo de deportistas, así como realizar una propuesta dietético-nutricional específica para los requerimientos de cada deporte en cada caso. Para el desarrollo del mismo, el alumnado trabajó en pequeños grupos y de forma colaborativa. Fueron los propios alumnos quiénes, en base a qué deporte realizaba el grupo de deportistas

(cc) EY-NC-ND 2018, Universitat Politècnica de València

Congreso IN-RED (2018) 
estudiado, eligieron aquellas pruebas de rendimiento deportivo que más información iban a dar sobre el deporte en cuestión.

Este problema, se enmarca dentro de la evaluación de la parte práctica de la asignatura, cuya evaluación se realizará de forma global, aunque se tendrá en cuenta todo el proceso llevado a cabo, coincidiendo con los diferentes aspectos que se evaluaron el curso anterior, están reflejados en la guía docente de la asignatura, y que son los siguientes: Resolución de casos de prácticas de problemas. Memorias, informes observación sistemática y cuaderno de notas de seminarios. Valoración nutricional de deportistas Trabajo bibliográfico realizado en pequeño grupo y exposición oral. Con ello, se pretendió completar los objetivos específos de la asignatura, que son: "Conocer las necesidades nutritivas y energéticas en diferentes situaciones de actividad física. Adquirir los conocimientos necesarios para realizar un balance energético equilibrado, para mantener y mejorar la salud a través de la práctica deportiva. Conocer y aplicar ténicas de evaluación nutricional, para la valoración de diferentes situaciones en la práctica deportiva."

\subsection{Evaluación}

En el proceso de recogida de datos se emplearon entrevistas iniciales, finales e informes de auto-seguimiento, siguiendo las indicaciones para estas según artículos de referencia para la evaluación de la innovación docente (Mauri, 2008).

Además de los datos recogidos en las entrevistas (antes y después de la implementación o no de la metodología ABP), se emplearon los datos de evaluación propios de la parte práctica de la asignatura, así como los datos de participación como asistentes o asistentes y ponentes en jornadas o congresos científicos.

\subsection{Análisis Estadístico}

Para la realización del análisis estadístico se empleó el software estadístico SPSS en su versión 24.0 para Mac (IBM Statistics, New York). Se llevaron a cabo estadísticos descriptivos (porcentajes o media \pm desviación estánar), prueba Kolmogorov-Sminornov, y comparación de medias mediante la prueba $\mathrm{T}$ para muestras independientes. Se fijó un nivel de significación de $\mathrm{p}<0,05$. Asimismo, se calculó el tamaño del efecto (TE) siguiendo las directrices de Cohen (1988). El TE se consideró despreciable $(<0,2)$, pequeño $(0,2-0,5)$, moderado $(0,5-0,8)$ y grande $(>0,8)$.

(cc) EY-NC-ND 2018, Universitat Politècnica de València 


\section{Resultados}

Los resultados del desarrollo de la innovación pusieron de manifiesto, en términos generales, una mayor participación del alumnado en relación a las tareas ofertadas. Así como un grado elevado de implicación con el trabajo de la asignatura, en el que se pone de manifiesto un interés explítico de la transferencia del trabajo en el contexto educativo hacia el ámbito profesional o de investigación, como son los congresos científicos. En la figura 1 se presentan los estadísticos descriptivos del alumnado durante los cursos 2016-2017 y 2017-2018. En esta se muestran los datos de la asistencia, por un lado, y presentación por parte del alumnado de comunicaciones científicas a congresos, por otro.

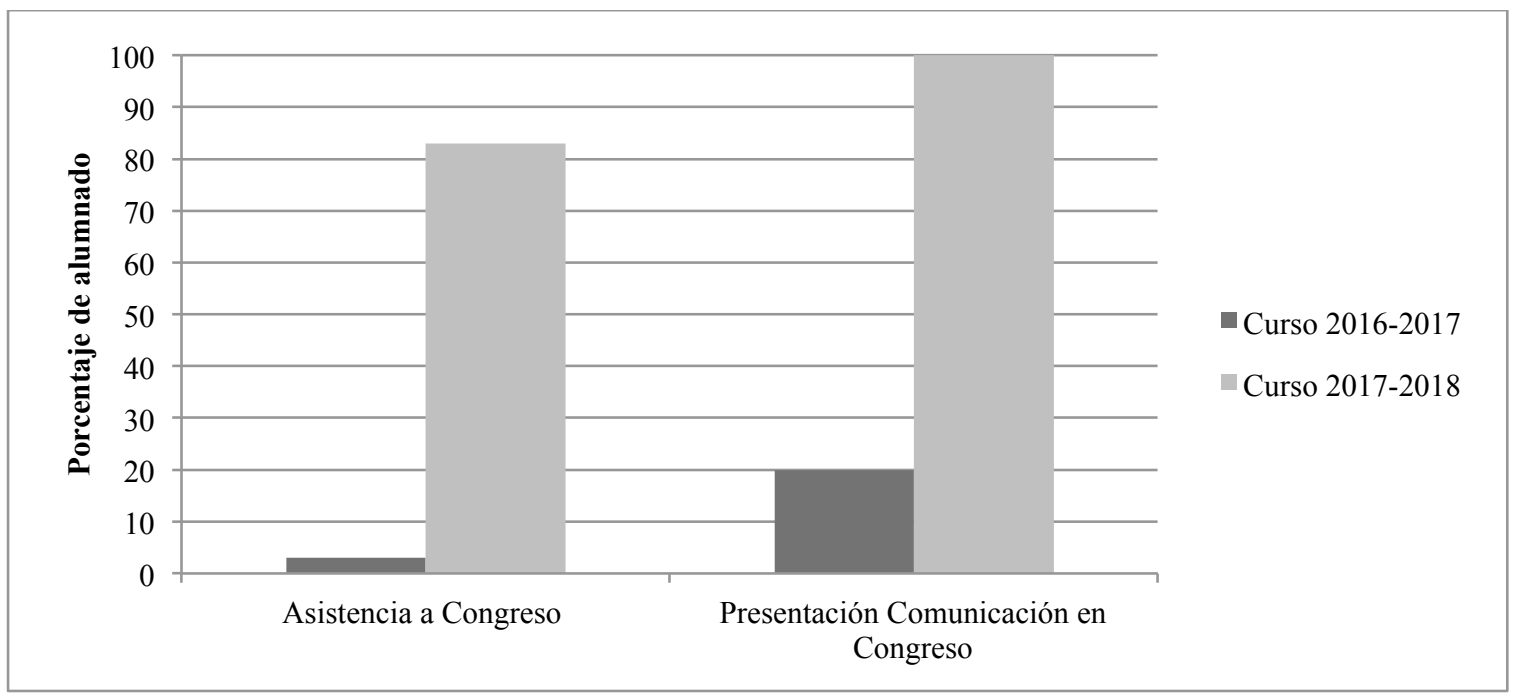

Fig. 1. Estadísticos descriptivos de asistencia a congresos y presentación de comunicaciones a congreso.

Asimismo, en la tabla 1 aparecen los resultados promedios de las calificaciones obtenidas por el alumnado en los cursos 2016-2017 y 2017-2018. Se pudo observar el alumnado del curso académico 2017-2018 obtuvieron calificaciones significativamente superiores, en comparación con el alumnado del curso previo, en la evaluación práctica de la asignatura, que también expresa un tamaño del efecto moderado-grande. 
Tabla 1. Resultados de la comparación de medias por curso académico de la evaluación práctica

\begin{tabular}{ccccc}
\hline Curso Académico & Estudiantes (n) & Evaluación Práctica & Significación & Tamaño Efecto (Cohen) \\
\hline $2016-2017$ & 30 & $7,0 \pm 0,7$ & $\mathrm{p}=0,040$ & 0,772 \\
$2017-2018$ & 35 & $7,5 \pm 0,6$ & \\
\hline
\end{tabular}

Además de la obtención de los estadísticos descriptivos y la realización de la comparación de ambos cursos, cabe destacar la importancia de los resultados obtenidos a través de las entrevistas iniciales y finales, después de la implementación ABP en ambos cursos.

En la entrevista inicial se abordaron cuestiones relacionadas con las características generales y específicas de la propuesta. Así como la distribución de las tareas por parte de los participantes, asumiendo ellos mismos sus responsabilidades. De forma inherente a la implementación educativa, se realizó la formación de los grupos, el diseño de la intervención a realizar, la valoración de la selección de los materiales (material antropométrico, balones medicinales, pulsómetros, etc.) a emplear, las actividades de evaluación que tendrán lugar, así como las expectativas de los resultados.

En el informe de seguimiento los grupos puso de manifiesto un adecuado desarrollo de las habilidades y destrezas sociales, actitudinales y cognitivas para la búsqueda, análisis, integración y transferencia de conocimientos. Además, los grupos aportaron las variaciones que habían realizado en relación con la propuesta inicial, así como la modificación parcial de algunos de los objetivos específicos propuestos en el caso del alumnado del curso 2017 2018. Mientras que el alumnado del curso anterior, no realizaron reajustes notables, ya que cada una de las sesiones se realizaron de forma guiada. Además, el alumnado del curso 2017-2018 aplicaron diversas variaciones, debido a la adaptación a las dificultades y obstáculos en las condiciones que se llevó a cabo la innovación. Asimismo, estos reflejaron un mayor interés en relación con la contribución al propio aprendizaje.

A través de la entrevista final, se identificó que en ambos casos ambos grupos del alumnado cumplieron los objetivos propuestos en la asignatura, así como se adquirieron las competencias planteadas en la guía docente de la asignatura. Cabe incidir en que la implicación, dedicación y el trabajo de calidad realizado, puso de manifiesto un interés elevado en la divulgación del trabajo científico por parte del alumnado en congresos específicos del área temática. Sin embargo, el alumnado del curso 2016-2017 mostraron un grado de satisfacción menor con el proceso de enseñanza-aprendizaje en comparación con los alumnos del curso 2017-2018. La valoración tanto de los elementos de la innovación como: las características organizativas, la cooperación, los aspectos metodológicos y

(cc) EY-NC-ND 2018, Universitat Politècnica de València 
didácticos y la evaluación, fueron mejores en el alumnado con el que se llevó a cabo la implementación de la metodología ABP.

Por ello, al mismo tiempo, el profesor refirió también implicaciones mayores en su preparación profesional. Debido a que durante el proceso debía hacer frente a nuevas actuaciones debido a la libertad de elección de intervenciones del alumnado, ya que durante el proceso también actuó como guía de apoyo en las situaciones más complejas o de difícil solución. resultado, el profesor acusará implicaciones en su preparación profesional, pues se le va a requerir, en suproceso de formación -inicial o de reciclaje-, ser usuarioaventajado de recursos de información. Junto a ello,necesitará servicios de apoyo de guías y ayudasprofesionales que le permitan participar enteramente enel ejercicio de su actividad.

\section{Conclusiones}

A modo de conclusión, cabe incidir en que la implementación metodológica del ABP has favorecido al desarrollo de las destrezas sociales, actitudinales y cognitivas para la búsqueda, análisis, integración y transferencia de conocimientos del alumando del Grado en Nutrición Humana y dietética. Con ello, el alumnado han desarrollado favorablemente estrategias y métodos que permitan ser aplicados y transferidos en el futuro a situaciones semejantes para la resolución de problemas. También, han demostrado la capacidad de auto-aprendizaje, trabajo en equipo y estimulación positiva del pensamiento crítco. Por último, destacar que los resultados obtenidos por el alumnado implementado con la metodología $\mathrm{ABP}$ mostraron mejores resultados en comparación con el alumnado no implementado con esta metodología.

\section{Referencias}

BERAZA, M. A. Z. (2013). "Innovación en la enseñanza universitaria". Contextos Educativos. Revista de Educación, (6), 113-136.

CUÉLLAR, A. I., Y ALONSO, M. I. (2010). "How evaluate the collaborative learning? A proposal valuing the process, content and the product of group interactivity". Revista General de Información y Documentación, 20, 221.

CABRA TORRES, F. (2008). "La evaluación y el enfoque de competencias: tensiones, limitaciones y oportunidades para la innovación docente en la universidad". Revista escuela de administración de negocios, (63).

COHEN J. (1988). Statistical Power Analysis for the Behavioral Sciences. 2nd ed. Hillsdale, NJ: Lawrence Erlbaum Associates.

ESTEBAN, C. M. R., \& Sánchez, C. M. (2005). "Innovación docente en la Universidad en el marco del EEES”. Educatio Siglo XXI, 23, 171-189.

(cc) EY-NC-ND 2018, Universitat Politècnica de València

Congreso IN-RED (2018) 
GARCÍA, C. M., RUÍZ, C. M., Y NOCHE, B. G. (2010). "Innovación educativa en España desde el punto de vista de sus protagonistas". Profesorado. Revista de Curriculum y Formación de Profesorado, 14(1), 111-134.

GÁlVEZ, I. E., REDRUELLO, R. A., MARTÍN, R. C., DE LA HERRÁN GASCÓN, A., DE MIGUEL BADESA, S., GARCÍA, M. G., ... E IZQUIERDO, R. M. R. (2007). "El aprendizaje basado en problemas como innovación docente en la universidad: posibilidades y limitaciones". Educación y futuro: Revista de investigación aplicada y experiencias educativas, (16), 85-100.

JOFRÉ, C., \& CONTRERAS, F. (2013). "Implementación de la metodología ABP (Aprendizaje Basado en Problemas) en estudiantes de primer año de la carrera de Educación Diferencial”. Estudios pedagógicos (Valdivia), 39(1), 99-113.

LÓPEZ PASTOR, V. M. (2008). "Desarrollando sistemas de evaluación formativa y compartida en la docencia universitaria. Análisis de resultados de su puesta en práctica en la formación inicial del profesorado". European Journal of Teacher Education, 31(3), 293311.

MAURI, T., COLL, C., Y ONRUBIA, J. (2008). "La evaluación de la calidad de los procesos de innovación docente universitaria. Una perspectiva constructivista". Revista de docencia universitaria, 1(1).

MICHAVILA, F. (2009). "La innovación educativa. Oportunidades y barreras". Arbor, 185(Extra), 3-8.

NOVAK, A. M., \& TREAGUST, D. F. (2017). “Adjusting claims as new evidence emerges: Do students incorporate new evidence into their scientific explanations?". Journal of Research in Science Teaching.

PEITEADO, M. G. (2013). "Los estilos de enseñanza y aprendizaje como soporte de la actividad docente". Journal of Learning Styles, 6(11).

SALINAS, J. (2004). "Innovación docente y uso de las TIC en la enseñanza universitaria". RUSC. Universities and Knowledge Society Journal, 1(1).

SINGER, L. M. Y ALEXANDER, P. A. (2017). "Reading on paper and digitally: What the past decades of empirical research reveal". Review of Educational Research, 87(6), 1007 1041 .

TIRADOS, R. M. G., Y MAURA, V. G. (2007). "Diagnóstico de necesidades y estrategias de formación docente en las universidades". Revista Iberoamericana de Educación,43(6), 6.

WARREN, C. A. (2017). "Empathy, Teacher Dispositions, and Preparation for Culturally Responsive Pedagogy”. Journal of Teacher Education, 0022487117712487. 